\title{
INHALTSVERZEICHNIS der Nummern 1-6 34. Jahrgang (2007)
}

\section{Artikel}

Apeltauer, Ernst: Sprachliche Frühförderung von Kindern mit Migrationshintergrund

Fiehler, Reinhard: Gesprochene Sprache - ein »sperriger « Gegenstand

Freudenfeld, Regina: Interkulturelle Moderation - Studium und Berufsaussichten für $\mathrm{DaF}$

Maijala, Minna: Was ein Lehrwerk können muss - Thesen und Empfehlungen zu Potenzialen und Grenzen des Lehrwerks im Unterricht Deutsch als Fremdsprache

Riemer, Claudia: DaF/DaZ und empirische Forschung: wechselnde Herausforderungen

Saengaramruang, Wanna: Deutschunterricht und Germanistikstudium in Thailand: früher, heute und morgen

$1,3-36$

$5,460-471$

$6,562-573$

$6,543-561$

$5,445-459$

$4,341-372$

\section{DaF im Ausland}

Jäger, Andreas; Jasny, Sabine: Zur Lage der Germanistik in Australien 2007 Jaeger, Daniel; Nied Curcio, Martina; Schlanstein, Lisa: Handlungsorientierter Deutschunterricht im dreijährigen Curriculum an italienischen Hochschulen

Lin-Liu, Hwei-ann: Entwicklungsgeschichte der deutschen Lieder. Eine Alternative zur Verbesserung des Verständnisses einer fremden Kultur

Vollstedt, Marina; Walter, Stephan: „Germanisten in die Wirtschaft«. Grundkenntnisse BWL, Fachsprache, interkulturelle Kompetenz und Berufsorientierung für Moskauer Philologiestudenten

Yang, Jianpei: Entwicklungsmerkmale der Fachsprachenvermittlung im chinesischen Deutschunterricht am Beispiel der Tongji-Universität

\section{Didaktik DaF / Aus der Praxis}

Döring, Sandra: Wer hat Angst vorm deutschen Verb? - Ein Projektvorschlag zur Verbstellung im Deutschen

Kara, Hannele: Mündliches Portfolio im DaF-Unterricht - ein Lehrexperiment anhand von Portfolioarbeiten

Lay, Tristan: »Sophie Scholl - Die letzten Tage«. Möglichkeiten zum Einsatz im DaF-Unterricht

Meyer, Almut: Fachfremdsprachenangebot zur deutschen Rechtssprache an der Rechtswissenschaftlichen Fakultät der Universität Turku

Neuland, Eva: Mündliche Kommunikation als Schlüsselkompetenz: Entwicklung eines Moduls für germanistische Studiengänge 
Pelka, Daniela: Regionalität im Oppelner Institut für Germanistik: Schlesienbezüge in Lehre und Forschung

$6,584-593$

Raith, Markus: »In der Ferne daheim«. Didaktische Überlegungen zum Verhältnis von Sprache, Musik und Identität

4, 403-408

Schmidt, Sabine; Schmidt, Karin: Erinnerungsorte - Deutsche Geschichte im DaF-Unterricht

$4,418-427$

Schröder, Jörg: E-Mail-Tutorium zur Unterstützung des Unterrichts »Schreiben«. Erfahrungen mit einem Gemeinschaftsprojekt zwischen Trier und Hangzhou/VR China

$5,487-502$

\section{Zur Diskussion gestellt}

Maitz, Péter; Elspaß, Stephan: Warum der »Zwiebelfisch « nicht in den Deutschunterricht gehört

Roggausch, Werner: Antwort auf Péter Maitz / Stephan Elspaß und Einladung zur Diskussion

\section{Tagungsankündigungen}

»Deutsch als fachbezogene Fremdsprache in Grenzregionen«. Internationale Konferenz vom 7.-8. Februar 2008 am Fremdsprachenzentrum der Wirtschaftsuniversität Bratislava

6,625

Internationaler wissenschaftlicher Kongress: »Deutsch als Fremdsprache aus internationaler Perspektive. Neuere Trends und Tendenzen « an der Zhejiang Universität, Hangzhou, VR China, vom 07. bis 09. September 2007

Jahreskongress Gesamtverband moderne Fremdsprachen (GMF) vom 27.-29. März 2008 in Leipzig: »Qualität entwickeln: Neue Wege in Unterricht und Lehrerbildung «

35. Jahrestagung des Fachverbands Deutsch als Fremdsprache (FaDaF): »Auf neuen Wegen. Deutsch als Fremdsprache in Forschung und Praxis« vom 31. Mai bis 02. Juni 2007 am Sprachenzentrum der FU Berlin

36. Jahrestagung des Fachverbands Deutsch als Fremdsprache (FaDaF): »daf integriert: literatur : medien : ausbildung « vom 24. bis 26. Mai 2008 in Düsseldorf

\section{Auswahlbibliographie/Rezensionen/Eingegangene Literatur}

Auswahlbibliographie von Neuerscheinungen für das Fach Deutsch als Fremdsprache 2006; zusammengestellt von Dietrich Eggers unter Mitarbeit von Dorothee Schwarck

»Für Sie gelesen«. Kommentare und Rezensionen zu fast hundert Neuerscheinungen für das Fach Deutsch als Fremdsprache, hrsg. von Lutz Köster unter Mitarbeit von Evelyn Müller-Küppers

Dissertationen und Magisterarbeiten DaF. Zusammengestellt und kommentiert von Fritz Neubauer 
Adamzik, Kirsten; Krause, Wolf-Dieter (Hrsg.): Text-Arbeiten. Textsorten im fremd- und muttersprachlichen Unterricht an der Schule und Hochschule. Tübingen: Narr, 2005 (Europäische Schriften zur Textlinguistik, 1) (Rossella Pugliese)

Aktaş, Ayfer: Die Semantik der deutschen Partikelverben sowie die Semantik und die Morphologie der von diesen abgeleiteten Substantiva. Frankfurt/M.: Lang, 2005 (Europäische Hochschulschriften: Reihe 1, Deutsche Sprache und Literatur, 1922) (Reinhard von Bernus)

Albrecht, Jörn: Übersetzung und Linguistik. Tübingen: Narr, 2005 (Grundlagen der Übersetzungsforschung, 2) (Ioana Balacescu; Bernd Stefanink)

Balhar, Susanne; Cyffka, Andreas; Quaas, Lydia: Pons Kompaktwörterbuch Deutsch als Fremdsprache. Stuttgart: Klett, 2005 (Karl-Walter Florin)

Balmus, Petra; Oebel, Guido; Reinelt, Rudolf (Hrsg.): Herausforderung und Chance. Krisenbewältigung im Fach Deutsch als Fremdsprache in Japan. Beiträge zur DaF-Werkstatt Westjapan, Ryukyu Universität, Okinawa, Japan, 12.-14. Dezember 2003. München: Iudicium, 2005 (Carsten Waychert)

Barr, David: ICT - Integrating Computers in Teaching. Creating a ComputerBased Language-Learning Environment. Frankfurt/M.: Lang, 2005 (Conny Bast)

Bartsch, Tim-Christian; Hoppmann, Michael; Rex, Bernd F.; Vergeest, Markus: Trainingsbuch Rhetorik. Paderborn: Schöningh, 2005 (Rhesis. Arbeiten zur Rhetorik und ihrer Geschichte 2; UTB 2689) (Susanne Duxa)

Bastani, Atousa; Ragg, Christine: Der Vorkurs. Einführung ins Deutsch-Lernen. Stuttgart: Klett, 2005 (Sabine Jentges)

Baumert, Andreas: Mit der Muttersprache auf Talfahrt. Die Frage nach der deutschen Identität. Paderborn: IFB, 2005 (Lesław Tobiasz)

Bausch, Karl-Richard; Burwitz-Melzer, Eva; Königs, Frank G.; Krumm, HansJürgen (Hrsg.): Aufgabenorientierung als Aufgabe. Arbeitspapiere der 26. Frühjahrskonferenz zur Erforschung des Fremdsprachenunterrichts. Tübingen: Narr, 2006 (Thomas Stahl)

Bogdal, Klaus-Michael (Hrsg.): Neue Literaturtheorien. Eine Einführung. 3. Auflage. Göttingen: Vandenhoeck \& Ruprecht, 2005 (Thomas Bleicher)

Bratschi, Rebekka: Xenismen in der Werbung. Die Instrumentalisierung des Fremden. Bern; Berlin; Brüssel; Frankfurt/M.; New York; Oxford; Wien: Lang, 2005 (FASK Publikationen des Fachbereichs Angewandte Sprach- und Kulturwissenschaft der Johannes-Gutenberg-Universität Mainz in Germersheim, Reihe A: Abhandlungen und Sammelbände 44) (Manuela von Papen)

Bunk, Gerhard J. S.: Phonetik aktuell. Kopiervorlagen mit 2 CD's. Ismaning: Hueber, 2005 (Seongho Son)

Büttner, Christian (Hrsg.): Lernen im Spiegel des Fremden. Konzepte, Methoden und Erfahrungen zur Vermittlung interkultureller Kompetenz. Frankfurt/M.: IKO - Verlag für Interkulturelle Kommunikation, 2005 (Birgit Sens)

Clément, Danièle: Syntaktisches Grundwissen. Eine Einführung für Deutschlehrer. Göttingen: Vandenhoeck \& Ruprecht, 2005 (Klaus Geyer)

Duxa, Susanne; Hu, Adelheid; Schmenk, Barbara (Hrsg.): Grenzen überschreiten. Menschen, Sprachen, Kulturen. Festschrift für Inge Christine Schwerdtfeger zum 60. Geburtstag. Tübingen: Narr, 2005 (Magdalena Pieklarz)

Eder, Ulrike: "Auf die mehrere Ausbreitung der teutschen Sprache soll fürgedacht werden «. Deutsch als Fremd- und Zweitsprache im Unterrichtssystem der Donaumonarchie zur Regierungszeit Maria Theresias und Josephs II. Innsbruck: Studienverlag, 2006 (Theorie und Praxis - Österreichische Beiträge zu Deutsch als Fremdsprache 9, Serie B) (Libuše Spačilová)

$2 / 3,126-129$

$2 / 3,129-131$

$2 / 3,131-136$

$2 / 3,136-139$

$2 / 3,139-142$

$2 / 3,142-143$

$2 / 3,143-145$

$2 / 3,146-148$

$2 / 3,148-150$

$2 / 3,150-152$

$2 / 3,152-154$

$2 / 3,154-157$

2/3, 157-159

2/3, 159-160

$2 / 3,161-164$

$2 / 3,164-168$

Faigle, Iris: Rundum. Einblicke in die deutsche Kultur. Lehrbuch und CD. Lehrerhandbuch. Stuttgart: Klett, 2006 (Sonja Rosenbrock) 
Faulstich, Werner: Die Kultur der 80er Jahre. Paderborn: Fink, 2005 (Kulturgeschichte des 20. Jahrhunderts) (Thomas Bleicher)

Fehling, Sylvia: Language Awareness und bilingualer Unterricht. Eine komparative Studie. Frankfurt/M.: Lang, 2005 (LaCuLi. Language Culture Literacy, 1) (Sabine Garsz)

Foschi Albert, Marina; Hepp, Marianne; Neuland, Eva (Hrsg.): Texte in Sprachforschung und Sprachunterricht. Pisaner Fachtagung 2004 zu neuen Wegen der italienisch-deutschen Kooperation. München: Iudicium, 2006 (Lucia Cinato)

Frank, Norbert; Stary, Joachim: Gekonnt visualisieren. Medien wirksam einsetzen. Paderborn: Schöningh, 2006 (UTB 2818) (Daniela Schäferkordt)

Fremdsprache Deutsch Heft 33: Lust auf Internet. Herausgegeben von Markus Biechele. Stuttgart: Klett, 2005 (Karl-Walter Florin)

Frey, Evelyn; Kort-Klimach, Iris; Kotz, Andreas; Lundquist-Mog, Angelika; Nordkämper-Schleicher, Ulrike; Zeisig, Nicole: Alles drin. Praxishandbuch Grundstufe Deutsch. Stuttgart: Klett, 2005 (Seongho Son)

Funke, Reinold: Sprachliches im Blickfeld des Wissens. Grammatische Kenntnisse von Schülerinnen und Schülern. Tübingen: Niemeyer, 2005 (Klaus Geyer)

Gilles, Peter: Regionale Prosodie im Deutschen. Variabilität in der Intonation von Abschluss und Weiterweisung. Berlin: de Gruyter, 2005 (Katja Reinecke)

Görner, Rüdiger (Hrsg.): Anglo-German Affinities and Antipathies. München: Iudicium, 2004 (Manuela von Papen)

Grammenou, Gaby: Hörtraining für die Mittelstufe. Übungsbuch 2. Auflage. Paket: Lehrbuch (inklusive Übungsbuch) und 12 CD's. Athen: KarabatosVerlag, 2005 (zu beziehen über den Hueber-Verlag, Ismaning) (Karl-Walter Florin)

Greiner, Norbert: Übersetzung und Literaturwissenschaft. Tübingen: Narr, 2004 (Grundlagen der Übersetzungsaforschung 1) (Ioana Balacescu; Bernd Stefanink)

Guckelsberger, Susanne: Mündliche Referate in universitären Lehrveranstaltungen. Diskursanalytische Untersuchungen im Hinblick auf eine wissenschaftsbezogene Qualifizierung von Studierenden. München: Iudicium, 2005 (Studien Deutsch, 34) (Joachim Schlabach)

Haberzettl, Stefanie: Der Erwerb der Verbstellungsregeln in der Zweitsprache Deutsch durch Kinder mit russischer und türkischer Muttersprache. Tübingen: Niemeyer, 2005 (Linguistische Arbeiten, 495) (Eva Sommer)

Harms, Michael: »TV is quite a big party of my life.« Konsequenzen aus der Dominanz von Bildlichkeit im Lerneralltag für den Fremdsprachenunterricht in Großbritannien. Regensburg: FaDaF, 2005 (Materialien Deutsch als Fremdsprache 72) (Stefan Rahn)

Haubner, Angela: Ausländische Inländerinnen - Migrantentöchter in der Postmoderne. Frankfurt/M.: IKO-Verlag für interkulturelle Kommunikation, 2005 (Eva Sommer)

Hellwig, Karlheinz: Bildung durch Literatur. Frankfurt/M.: Lang, 2005 (Fremdsprachendidaktik inhalts- und lernerorientiert, 10) (Karl Esselborn)

Hentig, Hartmut von: Wissenschaft. Eine Kritik. Weinheim: Beltz, 2005 (Ralph A. Hartmann)

Hernig, Markus: Deutsch als Fremdsprache. Eine Einführung. Wiesbaden: Verlag für Sozialwissenschaften, 2005 (Studienbücher zur Linguistik) (Werner Heidermann)

Hill-Zenk, Anja; Sousa, Karin (Hrsg.): To read or not to read. Von Leseerlebnissen und Leseerfahrungen. Leseförderung und Lesemarketing, Leselust und Lesefrust. München: Iudicium, 2004 (Publications of the Institute of Germanic Studies 83) (Sabine Jentges)

$2 / 3,174-176$

2/3, 176-178

5, 531-533

2/3, 178-182

2/3, 182-184

2/3, 184-185

$2 / 3,186-188$

2/3, 188-190

2/3, 190-192.

2/3, 192-193

2/3, 131-136

2/3, 193-195

2/3, 195-196

2/3, 196-198

2/3, 198-199

2/3, 199-201

2/3, 201-203

2/3, 203-206

2/3, 206-208 
Hinnenkamp, Volker; Meng, Katharina: Sprachgrenzen überspringen. Sprachliche Hybridität und polykulturelles Selbstverständnis. Tübingen: Narr, 2005 (Studien zur deutschen Sprache 32) (Sabine Grasz)

Hoffmann, Dieter: Arbeitsbuch deutschsprachige Prosa seit 1945. 2 Bände. Tübingen: Francke, 2006 (UTB 2731) (Karl Esselborn)

Hüllen, Werner: Kleine Geschichte des Fremdsprachenlernens. Berlin: Schmidt, 2005 (Conny Bast)

Jaeger, Friedrich; Liebsch, Burkhard; Rüsen, Jörn; Straub, Jürgen: Handbuch der Kulturwissenschaften. Gesamtwerk in 3 Bänden. Stuttgart: Metzler, 2004 (Wolfgang Braune-Steininger)

Japanische Gesellschaft für Germanistik (Hrsg.): Universale Syntax und Semantik für Sprachvergleiche. München: Iudicium, 2005 (Neue Beiträge zur Germanistik Band 4, Heft 2. Internationale Ausgabe von Doitsu Bungaku) (Markus J. Weininger)

Jungen, Oliver; Lohnstein, Horst: Einführung in die Grammatiktheorie. München: Fink, 2006 (UTB 2676) (Markus J. Weininger)

Jureit, Ulrike; Wildt, Michael (Hrsg.): Generationen. Zur Relevanz eines wissenschaftlichen Grundbegriffs. Hamburg: Hamburger Edition, 2005 (Manfred Kaluza)

Karg, Ina: Mythos PISA. Vermeintliche Vergleichbarkeit und die Wirklichkeit eines Vergleichs. Göttingen: V \& R unipress, 2005 (Ralph A. Hartmann)

Kaufmann, Susann; Rohrmann, Lutz; Szablewski-Cavus, Petra: Orientierungskurs Geschichte - Institutionen - Leben in Deutschland. Berlin: Langenscheidt, 2005 (Sabine Jentges)

Kessel, Katja; Reimann, Sandra: Basiswissen Deutsche Gegenwartssprache. Tübingen: Francke, 2005 (UTB 2704) (Lesław Tobiasz)

Knobloch, Clemens; Schaeder, Burkhard: Wortarten und Grammatikalisierung. Perspektiven in System und Erwerb. Berlin: de Gruyter, 2005 (Linguistik Impulse \& Tendenzen, 12) (Heiko Narrog)

Kubaczek, Martin; Tsuchiya, Masahiko (Hrsg.): »Bevorzugt beobachtet«. Zum Japanbild in der zeitgenössischen Literatur. München: Iudicium, 2005 (Thomas Stahl)

Kusnetsova, Nadeshda; Löschmann, Martin: Deutsch für Architekten. Fachtexte - Übungen - Wortschatz. Tomsk: Print Manufacture Publishers, 2006 (Dagmar Blei)

Langenscheidt Grundschulwörterbuch Deutsch. Berlin: Langenscheidt, 2005 (Lutz Köster)

Lege, Peter: Wer, was, wann, wo? Das D-A-CH-Landeskunde-Quiz. Fragen und Antworten zu Deutschland, Österreich und der Schweiz. Berlin: Langenscheidt, 2005 (Sabine Jentges)

Limbach, Jutta (Hrsg.): Ausgewanderte Wörter. Ismaning: Hueber, 2007 (Anneliese Stein-Meintker)

Lodewick, Klaus: TestDaF-Training Neu. Vorbereitung auf den Test Deutsch als Fremdsprache. Text- und Übungsbuch. Tipps und Lösungen. 2 CDs zu den Hörtexten und zur mündlichen Prüfung. Göttingen: Fabouda, 2004 (Tibor Vigh)

Lohde, Michael: Wortbildung des modernen Deutschen. Ein Lehr- und Übungsbuch. Tübingen: Narr, 2006 (Narr Studienbücher) (Uwe Durst)

Lübke, Diethard: Übungen zur neuen Rechtschreibung. Neubearbeitung. Ismaning: Hueber, 2006 (deutsch üben, 10) (Fritz Neubauer)

Ludewig, Petra: Korpusbasiertes Kollokationslernen. Computer-Assisted Language-Learning als prototypisches Anwendungsszenario der Computerlinguistik. Frankfurt/M.: Lang, 2005 (Sprache, Sprechen und Computer, 9) (Markus J. Weininger)

$2 / 3,208-210$

$2 / 3,210-212$

$2 / 3,213-214$

$2 / 3,214-216$

$2 / 3,217-218$

2/3, 218-220

2/3, 220-222

$2 / 3,222-224$

2/3, 224-226

2/3, 226-228

2/3, 228-230

2/3, 230-233

2/3, 233-235

2/3, 235-237

2/3, 237-239

2/3, 239-240

2/3, 241-243

2/3, 243-245

2/3, 245-247

2/3, 248-252 
Ludwig, Hans-Werner: Arbeitsbuch Lyrikanalyse. 5., erweiterte und aktualisierte Auflage. Tübingen: Francke, 2005 (UTB 2727) (Simone Schiedermair)

Maaß, Kurt-Jürgen (Hrsg.): Kultur und Außenpolitik. Handbuch für Studium und Praxis. Baden-Baden: Nomos, 2005 (Wolfgang Braune-Steininger)

Meese, Herrad: Radio D. Sprachkurs mit Hörtexten für Anfänger. Berlin: Langenscheidt, 2005 (Eva Sommer)

Mehlhorn, Grit (unter Mitarbeit von Karl-Richard Bausch, Tina Claußen, Beate Helbig-Reuter, Karin Kleppin): Studienbegleitung für ausländische Studierende an deutschen Hochschulen. Teil I: Handreichungen für Kursleiter zum Studienstrategien-Kurs. Teil II: Individuelle Lernberatung - Ein Leitfaden für die Beratungspraxis. München: Iudicium, 2005 (Manfred Kaluza)

Müller, Andreas P.: Sprache und Arbeit. Aspekte einer Ethnographie der Unternehmenskommunikation. Tübingen: Narr, 2006 (Forum für Fachsprachen-Forschung, 71) (Karl-Hubert Kiefer)

Müller, Natascha; Kupisch, Tanja; Schmitz, Katrin; Kantone, Katja: Einführung in die Mehrsprachigkeitsforschung. Deutsch, Französisch, Italienisch. Tübingen: Narr, 2006 (Lesław Tobiasz)

Müller, Peter O.: Fremdwortbildung. Theorie und Praxis in Geschichte und Gegenwart. Frankfurt/M.: Lang, 2005 (Dokumentation Germanistischer Forschung, 6) (Eva Sommer)

Mummert, Ingrid: Begegnungen mit »Gertrud « und »Elsa«. Mündliche und schriftliche Interpretation deutschsprachiger Literatur mit ausländischen Studierenden. Eine Studie. Baltmannsweiler: Schneider Verlag Hohengehren, 2006 (Perspektiven Deutsch als Fremdsprache, 19) (Michaela Haberkorn)

Neuland, Eva (Hrsg.): Jugendsprachen - Spiegel der Zeit. Internationale Fachkonferenz 2001 an der Bergischen Universität Wuppertal. Frankfurt/M.: Lang, 2005 (Sprache - Kommunikation - Kultur. Soziolinguistische Beiträge, 2) (Wolfgang Braune-Steininger)

Neuland, Eva; Ehlich, Konrad; Roggausch, Werner (Hrsg.): Perspektiven der Germanistik in Europa. Tagungsbeiträge. München: Iudicium, 2005 (Wolfgang Braune-Steininger)

Nies, Fritz (Hrsg.): Europa denkt mehrsprachig. Exemplarisch: deutsche und französische Kulturwissenschaften. L'Europe pense en plusieurs Langues. Exemplaires: les Sciences de l'Homme en France et en Allemagne. Tübingen: Narr, 2005 (Transfer, 18. Düsseldorfer Materialien zur Literaturübersetzung) (Bernd Stefanink)

Ogawa, Akio: Dativ und Valenzerweiterung. Syntax, Semantik und Typologie. Tübingen: Stauffenburg, 2005 (Studien zur deutschen Grammatik, 66) (Heiko Narrog)

O'Reilly, Claire; Arnold, Maik: Interkulturelles Training in Deutschland. Theoretische Grundlagen; Zukunftsperspektiven und eine annotierte Literaturauswahl. Frankfurt/M.: IKO-Verlag für Interkulturelle Kommunikation, 2005 (Ellen Tichy)

Perlmann-Balme, Michaela; Schalb, Susanne; Weers, Dörte: em neu Brückenkurs. Kursbuch. Arbeitsbuch. Lehrerhandbuch. 2 Audio-CDs. 2 Kassetten. Ismaning: Hueber, 2006 (Kathrin Schweiger)

Plisch de Vega, Stefanie; Schurig, Cordula: Alltag in Deutschland. Materialien mit Übungen. Stuttgart: Klett, 2005 (Seongho Son)

Pupp Spinassé, Karen: Deutsch als Fremdsprache in Brasilien. Eine Studie über kontextabhängige unterschiedliche Lernersprachen und muttersprachliche Interferenzen. Frankfurt/M.: Lang, 2005 (Werkstattreihe Deutsch als Fremdsprache, 79) (Katja Reinecke)

Rapti, Aleka (Hrsg.): Entwicklung der Textkompetenz griechischer, in Deutschland aufgewachsener Kinder. Frankfurt/M.: Lang, 2005 (Birgit Sens) 
Reichmann, Tinka: Satzspaltung und Informationsstruktur im Portugiesischen und im Deutschen. Ein Beitrag zur kontrastiven Linguistik und Übersetzungswissenschaft. Frankfurt/M.: Lang, 2005 (Saarbrücker Beiträge zur Sprach- und Translationswissenschaft, 6) (Thomas Johnen)

Riegel, Christine: Im Kampf um Zugehörigkeit und Anerkennung. Orientierungen und Handlungsformen von jungen Migrantinnen. Eine soziobiografische Untersuchung. Frankfurt/M.: IKO-Verlag für Interkulturelle Kommunikation, 2005 (Beate Herberich)

Roche, Jörg: Fremdsprachenerwerb - Fremdsprachendidaktik. Tübingen: Francke, 2005 (Karin Madlener)

Röhner, Charlotte (Hrsg.): Erziehungsziel Mehrsprachigkeit. Diagnose von Sprachentwicklung und Förderung von Deutsch als Zweitsprache. Weinheim: Juventus, 2005 (Sigrid Luchtenberg)

Roth, Marita: Stereotype in gesprochener Sprache. Narrative Interviews mit Ostund Westberliner Sprechern 1993-1996 (Stauffenburg Linguistik, 36). Tübingen: Stauffenburg, 2005 (Sabine Ylönen)

Schemann, Hans: Bild - Sprachbild - Weltbild - Phantasiebild, Zur Natur des Bildes und seiner Beziehung zu Wort, Idee und Begriff. Hildesheim: Olms, 2005 (Germanistische Linguistik Monographien, 16) (Karl-Hubert Kiefer)

Schilling, Klaus von: Das politisch-soziale System der Bundesrepublik Deutschland. Ein Landeskunde-Kompendium. Teil I: Demokratisches Selbstverständnis, politische Institutionen und Rechtssystem. Köln: Saxa, 2006 (Skripten Interkulturelle Germanistik, 1) (Christine Kramel)

Schwitalla, Johannes; Wegstein, Werner (Hrsg.): Korpuslinguistik deutsch: synchron - diachron - kontrastiv. Würzburger Kolloquium 2003. Tübingen: Niemeyer, 2005 (Elźbieta Sierosławska)

Sick, Bastian: Der Dativ ist dem Genitiv sein Tod. Folge 3. Noch mehr Neues aus dem Irrgarten der deutschen Sprache. Köln: Kiepenheuer \& Witsch, 2006 (KiWi, 958) (Svenja Hammer)

Stolze, Radegundis: Übersetzungstheorien. Eine Einführung. 4. überarbeitete Auflage. Tübingen: Narr, 2005 (Narr Studienbücher) (Ioana Balacescu; Bernd Stefanink)

Takashi, Narita; Ogawa, Akio; Oya, Toshiaki (Hrsg.): Deutsch aus ferner Nähe. Japanische Einblicke in eine fremde Sprache. Festschrift für Susumu Zaima zum 60. Geburtstag. Tübingen: Stauffenburg, 2005 (Conny Bast)

Thalmayr, Andreas: Heraus mit der Sprache! Ein bisschen Deutsch Deutsche, Österreicher, Schweizer und andere Aus- und Inländer. München: Hanser, 2005 (Christian F. Arsan)

Thörle, Britta: Fachkommunikation im Betrieb. Interaktionsmuster und berufliche Identität in französischen Arbeitsbesprechungen. Tübingen: Narr, 2005 (Forum für Fachsprachen-Forschung, 70) (Thomas Johnen)

Ueding, Gert (Hrsg.): Rhetorik. Begriff - Geschichte - Internationalität. Tübingen: Niemeyer, 2005 (Elźbieta Sierosławska)

Vater, Heinz: Referenz-Linguistik. München: Fink, 2005 (UTB 2685) (Markus J. Weininger)

Wangerin, Wolfgang (Hrsg.): Musik und Bildende Kunst im Deutschunterricht. Baltmannsweiler: Schneider Verlag Hohengehren, 2006 (Lutz Köster)

Welke, Klaus: Tempus im Deutschen. Rekonstruktion eines semantischen Systems. Berlin: de Gruyter, 2006 (Linguistik - Impulse und Tendenzen 13) (Klaus Geyer)

Wiegand, Herbert Ernst (Hrsg.): Untersuchungen zur kommerziellen Lexikographie der deutschen Gegenwartssprache II. »Duden. Das große Wörterbuch der deutschen Sprache in zehn Bänden«. Print- und CD-ROM-Version. Band 2. Tübingen: Niemeyer, 2005 (Lutz Köster)

$2 / 3,288-291$

$2 / 3,291-293$

2/3, 293-295

2/3, 295-297

$2 / 3,297-300$

$2 / 3,300-302$

$2 / 3,302-304$

$2 / 3,304-306$

2/3, 306-307

2/3, 307-308

$2 / 3,308-310$

2/3, 310-313

2/3, 313-315

2/3, 316-317

$2 / 3,317-320$

2/3, 321-324

2/3, 324-326

2/3, 326-329 
Wierlacher, Alois; Ehlich, Konrad; Eichinger, Ludwig M.; Kelletat, Andreas F.; Krumm, Hans-Jürgen; Michel, Willy (Hrsg.): Jahrbuch Deutsch als Fremdsprache. Intercultural German Studies. Band 30: Sprachenpolitik. München: Iudicium, 2004 (Katalin Petneki)

$2 / 3,329-331$

Wolff, Armin; Riemer, Claudia; Neubauer, Fritz: Sprache lehren - Sprache lernen. Beiträge der 32. Jahrestagung des Fachverbandes Deutsch als Fremdsprache 2004 in Bielefeld. Regensburg: FaDaF, 2005 (Materialien Deutsch als Fremdsprache, 74) (Dorothea Spaniel-Weise)

Wuttke, Evelyne: Unterrichtskommunikation und Wissenserwerb. Zum Einfluss von Kommunikation auf den Prozess der Wissensgenerierung. Frankfurt/M.: Lang, 2005 (Konzepte des Lehrens und Lernens, 11) (Joachim Schlabach)

$2 / 3,331-334$

$2 / 3,334-336$

Zwischenstaatliche Kommission für deutsche Rechtschreibung (Hrsg.): Deutsche Rechtschreibung: Regeln und Wörterverzeichnis. Amtliche Regelung. Tübingen: Narr, 2005 (Fritz Neubauer)

$2 / 3,336-337$

\section{Stichwortregister}

Australien (Germanistik 2007) 5, 472-486

Auswahlbibliographie von Neuerscheinungen 1, 82-111

Berufsaussichten (Studiengang Interkulturelle Moderation) 6, 562-573

Berufsorientierung von Moskauer Philologiestudenten 1,37-53

BWL (Fachsprache Wirtschaft) 1, 37-53

chinesischer Deutschunterricht 4, 373-389

E-Mail-Tutorium im Unterricht "Schreiben « $5,487-502$

empirische Forschung DaF/DaZ 5, 445-459

Deutsch als Fremdsprache

Australien (Germanistik 2007) 5, 472-486

Auswahlbibliographie von Neuerscheinungen 1, 82-111

Berufsaussichten (Studiengang Interkulturelle Moderation) 6, 562-573

Berufsorientierung von Moskauer Philologiestudenten 1,37-53

chinesischer Deutschunterricht 4, 373-389

deutsche Geschichte im DaF-Unterricht (Erinnerungsorte) 4, 418-427

deutsche Rechtssprache (Fachsprachenangebot an der Universität Turku) 4, 409417

Deutschunterricht in Thailand 4,341-372

Deutschunterricht an italienischen Hochschulen (handlungsorientiert) 4, 390402

Dissertationen und Magisterarbeiten (kommentiert) 6, 594-620

E-Mail-Tutorium im Unterricht »Schreiben « 5, 487-502 empirische Forschung DaF/DaZ 5, 445459

DaZ: empirische Forschung 5, 445-459

Erinnerungsorte (deutsche Geschichte im DaF-Unterricht) 4, 418-427

Fachsprache Recht an der Universität Turku 4, 409-417

Fachsprache Wirtschaft (Grundkenntnisse BWL) 1, 37-53

Fachsprache und Deutschunterricht in China $4,373-389$

Film im DaF-Unterricht (»Sophie Scholl Die letzten Tage «) 5,503-514

Germanisten in der Wirtschaft 1,37-53

Germanistik in Australien 5, 460-486

Germanistikstudium in Thailand 4, 341372

gesprochene Sprache $\quad 5,460-471$

Grenzen und Potenziale von DaF-Lehrwerken 6,543-561

Hangzhou/VR China (Gemeinschaftsprojekt "Schreiben« als Unterrichtsprojekt mit E-Mail mit der Universität Trier 5, 487-502

Interkulturelle Moderation (als Studiengang) 6, 562-573

italienische Hochschulen (handlungsorientierter Deutschunterricht) 4, 390-402

Lehrwerke für DaF (Was sie können müssen) $6,543-561$

Lieder als Alternative zum Verständnis fremder Kultur 6,574-583

Migrantenhintergrund von Kindern (sprachliche Förderung) 1, 3-36 
Moskauer Philologiestudenten (Berufsorientierung) 1,37-53

mündliche Kommunikation als Schlüsselkompetenz 4, 428-437

mündliches Portfolio im DaF-Unterricht 1, 62-75

Oppeln (Institut für Germanistik) 6, 584593

Portfolioarbeiten 1, 62-75

Potenziale und Grenzen von DaF-Lehrwerken 6, 543-561

Kinder mit Migrantenhintergrund 1, 3-36

Schlesienbezüge in Forschung und Lehre an Institut für Germanistik in Oppeln 6, 584-593

Schlüsselkompetenz mündliche Kommunikation 4, 428-437

"Schreiben« als Unterrichtsprojekt mit EMail 5, 487-502

»Sophie Scholl - Die letzten Tage« (Film im DaF-Unterricht) 5, 503-514

Sprachliche Frühförderung von Kindern (mit Migrantenhintergrund) 1, 3-36

Studium und Berufsaussichten (Studiengang Interkulturelle Moderation) 6, 562-573

Thailand (Deutschunterricht und Germanistikstudium) 4, 341-372

Universität Trier (Gemeinschaftsprojekt »Schreiben « als Unterrichtsprojekt mit EMail mit der Universität Hangzhou/VR China 5, 487-502

Universität Turku (Fachsprachenangebot: Fachsprache Recht) 4, 409-417

Unterrichtsprojekt "Schreiben « mit einem E-Mail-Tutorium 5, 487-502

Verbstellung im Deutschen 1,54-61

DaF-Lehrwerke (Potenziale und Grenzen) 6, 543-561

»Der Dativ ist dem Genitiv sein Tod « (Bastian Sick zur Diskussion gestellt) 5, 515-526 und 5, 527-530

deutsche Geschichte im DaF-Unterricht (Erinnerungsorte) 4, 418-427

deutsche Rechtssprache (Fachsprachenangebot an der Universität Turku) 4, 409-417

deutsches Lied (Entwicklungsgeschichte) 6, 574-583

Deutschunterricht an italienischen Hochschulen 4, 390-402

Deutschunterricht in Thailand 4,341-372

Dissertationen und Magisterarbeiten (kommentiert) 6, 594-620 dreijähriges Curriculum an italienischen Hochschulen 4,390-402

Entwicklungsgeschichte des deutschen Liedes $6,574-583$

Erinnerungsorte (deutsche Geschichte im DaF-Unterricht) 4, 418-427

Fachsprache Recht an der Universität Turku 4, 409-417

Fachsprache Wirtschaft (Grundkenntnisse BWL) 1,37-53

Fachsprachenvermittlung im chinesischen Deutschunterricht 4,373-389

Film im DaF-Unterricht (»Sophie Scholl - Die letzten Tage «) 5,503-514

Germanistische Studiengänge (Schlüsselkompetenz mündliche Kommunikation) 4, 428-437

Germanisten in der Wirtschaft 1,37-53

Germanistik in Australien 5, 472-486

Germanistikstudium in Thailand 4, 341-372

gesprochene Sprache 5, 460-471

Grenzen und Potenziale von DaF-Lehrwerken 6, 543-561

handlungsorientierter Deutschunterricht an italienischen Hochschulen 4, 390-402

Hangzhou/VR China (Gemeinschaftsprojekt "Schreiben« als Unterrichtsprojekt mit EMail mit der Universität Trier 5, 487-502

Interkulturelle Moderation (als Studiengang) $6,562-573$

italienische Hochschulen (handlungsorientierter Deutschunterricht) 4, 390-402

Kinder mit Migrantenhintergrund 1, 3-36

Lehrwerke für $\mathrm{DaF}$ (was sie können müssen) 6, 543-561

Lieder als Alternative zum Verständnis fremder Kultur 6, 574-583

Migrantenhintergrund von Kindern (sprachliche Förderung) 1,3-36

Modul Schlüsselkompetenz mündliche Kommunikation (in germanistischen Studiengängen) 4, 428-437

Moskauer Philologiestudenten (Berufsorientierung) 1,37-53

mündliche Kommunikation als Schlüsselkompetenz 4, 428-437

mündliches Portfolio im DaF-Unterricht 1, 62-75

Oppeln (Institut für Germanistik) 6, 584-593

Portfolioarbeiten 1,62-75

Potenziale und Grenzen von DaF-Lehrwerken 6, 543-561 
Schlesienbezüge in Forschung und Lehre am Institut für Germanistik in Oppeln 6,584593

Schlüsselkompetenz mündliche Kommunikation 4, 428-437

"Schreiben « als Unterrichtsprojekt mit E-Mail 5, 487-502

Sick, Bastian zur Diskussion gestellt (»Der Dativ ist dem Genitiv sein Tod «) 5,515-526 und 5, 527-530

»Sophie Scholl - Die letzten Tage« (Film im DaF-Unterricht) 5, 503-514

Sprache, Musik und Identität (didaktische Überlegungen zu ihrem Verhältnis zueinander) 4, 403-408

Sprachen- und Dolmetscher-Institut München (Studiengang Interkulturelle Moderation) $6,562-573$

Sprachliche Frühförderung von Kindern (mit Migrantenhintergrund) 1,3-36

\section{Autorenverzeichnis}

Apeltauer, Ernst 1,3-36

Arsan, Christian F. 2/3, 310-313

Balacescu, Ioana 2/3, 131-136; 2/3, 307-308

Bast, Conny 2/3, 142-143; 2/3, 213-214; 2/3, 308-310

Bernus, Reinhard von 2/3, 129-131

Blei, Dagmar 2/3, 233-235

Bleicher, Thomas 2/3, 152-154; 2/3, 174-176

Braune-Steininger, Wolfgang $2 / 3,214-216$; $2 / 3,255-256 ; 2 / 3,270-272 ; 2 / 3,273-275$

Cinato, Lucia $5,531-533$

Döring, Sandra 1,54-61

Durst, Uwe 2/3, 243-245

Duxa, Susanne 2/3, 143-145

Eggers, Dietrich 1,82-111

Elspaß, Stephan 5, 515-526

Esselborn, Karl 2/3, 199-201; 2/3, 210-212

Fiehler, Reinhard 5, 460-471

Florin, Karl-Walter $\quad 2 / 3,136-139 ; 2 / 3,182-$ $184 ; 2 / 3,192-193$

Freudenfeld, Regina $\quad 6,562-573$

Grasz, Sabine 2/3, 176-178; 2/3, 208-210

Geyer, Klaus 2/3,161-164;2/3, 186-188;2/3, 324-326

Haberkorn, Michaela 2/3, 267-270

Hammer, Svenja 2/3, 306-307

Hartmann, Ralph A. 2/3, 201-203; 2/3, 222224

Heidermann, Werner 2/3, 203-206
Studium und Berufsaussichten (Studiengang Interkulturelle Moderation) 6, 562-573

Thailand (Deutschunterricht und Germanistikstudium) 4, 341-372

Tongji-Universität (Fachsprachenvermittlung und Deutschunterricht) 4, 373-389

Universität Oppeln (Schlesienbezüge in Forschung und Lehre) 6, 584-593

Universität Trier (Gemeinschaftsprojekt "Schreiben" als Unterrichtsprojekt mit EMail mit der Universität Hangzhou/VR China 5, 487-502

Universität Turku (Fachsprachenangebot: Fachsprache Recht) 4, 409-417

Unterrichtsprojekt "Schreiben" mit einem EMail-Tutorium 5, 487-502

Verhältnis von Sprache, Musik und Identität (didaktische Überlegungen) 4, 403-408

Verbstellung im Deutschen 1,54-61

Herberich, Beate 2/3, 291-293

Jäger, Andreas 5, 472-486

Jaeger, Daniel 4, 390-402

Jasny, Sabine 5, 472-486

Jentges, Sabine 2/3, 146-148; 2/3, 206-208; $2 / 3,224-226 ; 2 / 3,237-239$

Johnen, Thomas 2/3, 288-291; 2/3, 313-315

Kaluza, Manfred 2/3, 220-222; 2/3, 250-260

Kara, Hannele 1, 62-75

Kiefer, Karl-Hubert $\quad 2 / 3,260-263 ; 2 / 3,300-$ 302

Köster, Lutz 2/3, 235-237; 2/3, 321-324; 2/3, 326-329

Kramel, Christine $\quad 2 / 3,303-304$

Lay, Tristan 5, 503-314

Lin-Liu, Hwei-ann 6, 574-583

Luchtenberg, Sigrid 2/3, 295-297

Madlener, Karin 2/3, 293-295

Maijala, Minna 6, 543-561

Maitz, Péter 5, 515-526

Meyer, Almut 4, 409-417

Narrog, Heiko $2 / 3,228-230 ; 2 / 3,277-279$

Neubauer, Fritz 2/3, 245-247; 2/3, 336-337; 6, 594-620

Neuland, Eva 4, 428-438

Nied Curcio, Martina 4,390-402

Papen, Manuela von 2/3, 154-157; 2/3, 190192

Pelka, Daniela $\quad 6,584-593$ 
Petneki, Katalin 2/3, 329-331

Pieklarz, Magdalena 2/3, 164-168

Pugliese, Rossella 2/3, 126-129

Rahn, Stefan 2/3, 196-198

Raith, Markus 4, 403-408

Reinecke, Katja $\quad 2 / 3,188-190 ; 2 / 3,285-286$

Riemer, Claudia 5, 445-459

Roggausch, Werner 5,527-530

Rosenbrock, Sonja 2/3, 171-174

Saengaramruang, Wanna 4,341-372

Schäferkordt, Daniela $2 / 3,178-182$

Schiedermair, Simone 2/3, 252-255

Schlabach, Joachim 2/3, 193-195; 2/3, 334336

Schlanstein, Lisa $\quad 4,390-402$

Schmidt, Karin 4, 418-427

Schmidt, Sabine $\quad 4,418-427$

Schröder, Jörg 5, 487-502

Schwarck, Dorothee 1,82-111

Schweiger, Kathryn 2/3, 281-283

Sens, Birgit $2 / 3,159-160 ; 2 / 3,287-288$

Sierosławska, Elźbieta 2/3, 304-306; 2/3, 316-317
Sommer, Eva $\quad 2 / 3,195-196 ; 2 / 3,198-199 ; 2$ / $3,256-258 ; 2 / 3,265-267$

Son, Seongho $\quad 2 / 3,157-159 ; 2 / 3,184-185 ; 2$ / 3, 283-285

Spačilová, Libuše $\quad 2 / 3,168-171$

Spaniel-Weise, Dorothea 2/3, 331-334

Stahl, Thomas $2 / 3,150-152 ; 2 / 3,230-233$

Stefanink, Bernd 2/3, 131-136; 2/3, 275-277

Stein-Meintker, Anneliese 2/3, 239-240

Tichy, Ellen 2/3, 279-280

Tobiasz, Lesław 2/3, 148-150; 2/3, 226-227; $2 / 3,263-265$

Vigh, Tibor $2 / 3,241-243$

Vollstedt, Marina 1,37-53

Walter, Stephan $1,37-53$

Waychert, Carsten 2/3, 139-142

Weininger, Markus J. 2/3, 217-218; 2/3, 218$220 ; 2 / 3,248-252 ; 2 / 3,317-320$

Wolff, Armin 6, 629-639

Yang, Jianpei 4, 373-389

Ylönen, Sabine $\quad 2 / 3,297-300$

(zusammengestellt von Armin Wolff, Regensburg) 